\title{
Cytoplasmic dynein participates in meiotic checkpoint inactivation in mouse oocytes by transporting cytoplasmic mitotic arrest-deficient (Mad) proteins from kinetochores to spindle poles
}

\author{
Dong Zhang, Shen Yin, Man-Xi Jiang, Wei Ma, Yi Hou, Cheng-Guang Liang, Ling-Zhu Yu, \\ Wei-Hua Wang and Qing-Yuan Sun \\ State Key Laboratory of Reproductive Biology, Institute of Zoology, Chinese Academy of Sciences, Beijing 100080,
} China

Correspondence should be addressed to Q-Y Sun; Email: sunqy@ioz.ac.cn

D Zhang and S Yin contributed equally to this work

\begin{abstract}
The present study was designed to investigate the localization and function of cytoplasmic dynein (dynein) during mouse oocyte meiosis and its relationship with two major spindle checkpoint proteins, mitotic arrest-deficient (Mad) 1 and Mad2. Oocytes at various stages during the first meiosis were fixed and immunostained for dynein, Mad1, Mad2, kinetochores, microtubules, and chromosomes. Some oocytes were treated with nocodazole before examination. Anti-dynein antibody was injected into the oocytes at germinal vesicle (GV) stage before the examination of its effects on meiotic progression or Mad1 and Mad2 localization. Results showed that dynein was present in the oocytes at various stages from GV to metaphase II and the locations of Mad1 and Mad2 were associated with dynein's movement. Both Mad1 and Mad2 had two existing states: one existed in the cytoplasm (cytoplasmic Mad1 or cytoplasmic Mad2), which did not bind to kinetochores, while the other bound to kinetochores (kinetochore Mad1 or kinetochore Mad2). The equilibrium between the two states varied during meiosis and/or in response to the changes of the connection between microtubules and kinetochores. Cytoplasmic Mad1 and Mad2 recruited to chromosomes when the connection between microtubules and chromosomes was destroyed. Inhibition of dynein interferes with cytoplasmic Mad1 and Mad2 transportation from chromosomes to spindle poles, thus inhibits checkpoint silence and delays anaphase onset. These results indicate that dynein may play a role in spindle checkpoint inactivation.
\end{abstract}

Reproduction (2007) 133 685-695

\section{Introduction}

In mitotic cells, accurate chromosome segregation is ensured by the intrinsic feature of mitotic apparatus and the efficient regularity of spindle checkpoint system (Maney et al. 1999). The spindle checkpoint system is a highly conservative signal pathway and controls the anaphase onset with spindle integrity and corrects chromosome orientation in the spindle (Maney et al. 1999, Hoyt 2001, Wang \& Sun 2006). Any abnormality on the attachment between kinetochores and kinetochore-associated microtubules can activate the checkpoint, which in turn inhibits the anaphase-promoting complex and keeps cell arrest at the metaphase stage (Maney et al. 1999). The key interaction between chromosomes and microtubules is kinetochores.
Kinetochores possess at least three functions: attaching chromosomes to the mitotic spindle, controlling chromosome movement, and maintaining the mitotic spindle checkpoint (Maney et al. 1999). If kinetochores on unattached chromosomes were destroyed, the cell could overcome the metaphase arrest and progress into the anaphase (Rieder \& Salmon 1998). Unattached kinetochores serve as special sites and microenvironment for the organization of checkpoint proteins and then produce 'anaphase-waiting' signal (Rieder \& Salmon 1998, Maney et al. 1999). Many proteins, including mitotic arrest-deficient protein (Mad) 1, Mad2, Mad3, budding uninhibited by benzimidazole (Bub) 1, Bub2, Bub3 and others, participate in checkpoint pathway (Hoyt et al. 1991, Li \& Murray 1991, Roberts et al. 1994, Weiss \& Winey 1996, Shannon et al. 2002, Tsurumi et al. 
2004, Vigneron et al. 2004). Although checkpoint pathway in mitosis has been widely studied, the exact signal transduction is far more complicated and not well understood.

Recent studies conducted by us and other researchers have revealed that some mitotic spindle checkpoint proteins, such as Mad2 (Kallio et al. 2000, Shannon et al. 2002, Wassmann et al. 2003, Zhang et al. 2004, Homer et al. 2005, Ma et al. 2005), Mad1 (Zhang et al. 2005) and Bub1 (Shannon et al. 2002, Brunet et al. 2003,) were also found to function in meiosis. These studies indicate that Mad2 and, possibly, Mad1 participate in meiotic spindle checkpoint. We also found that checkpoint proteins are transported between cytoplasm and kinetochores in the oocytes depending on cell cycle stages and microtubule connection to chromosomes (Zhang et al. 2004, 2005). For example, both Mad1 and Mad2 mainly bind to kinetochores in the oocytes at prometaphase in which meiotic spindle has not formed and chromosomes have not aligned at the spindle. However, when the oocytes reach metaphase and all chromosomes have aligned at the spindle equator, both Mad1 and Mad2 are depleted from kinetochores and move to the spindle poles. If the microtubule-disassembly drug, nocodazole, is used to disassemble the metaphase spindle, both Mad1 and Mad2 move back to the chromosomes and re-bind to kinetochores (Zhang et al. 2004, 2005). Through this checkpoint pathway, checkpoint proteins control the anaphase onset. These results also indicate that there are some motor protein(s) in the oocytes that is responsible for transporting the checkpoint proteins between the kinetochores and the cytoplasm.

Cytoplasmic dynein (or dynein), a minus-end-directed microtubule motor, has been found to participate in microtubule attachment and other cellular and developmental processes, including transportation of some organelles and proteins (Pfarr et al. 1990, Escheverri et al. 1996, King et al. 2000, Howell et al. 2001, Sun \& Schatten 2006). It has been reported that prometaphase kinetochores had more dynein signals than metaphase kinetochores in mitotic cells, and dynein was recruited to metaphase kinetochores after depolymerization of microtubules (Pfarr et al. 1990, Escheverri et al. 1996). Dynein is also very sensitive to the attachment of microtubules to kinetochores, once the attachment starts in the early stage during mitosis, most of the dynein detach from kinetochores (King et al. 2000). In Drosophila, inhibition of the expression of ZW10 or ROD, which were in charge of dynein localization to kinetochores (Starr et al. 1998, Scaerou et al. 1999) caused the significant decrease in chromosome poleward movement, indicating that dynein at the kinetochores was involved in chromosome movement (Savoian et al. 2000, Sharp et al. 2000). Inhibition (Vaisberg et al. 1993) or overexpression of dynein (Echeverri et al. 1996) inhibited the spindle assembly, indicating that dynein also has function in mitotic spindle assembly. Dynein also participates in mitotic spindle checkpoint (Howell et al. 2001). For example, in Ptkl cells, microinjection of anti-dynein antibody prevented Mad2, Bub1, centromere protein $\mathrm{E}$ (CENP-E), and 3F3/2 phosphoantigen from transporting to the spindle poles, prevented Mad2 detachment from the kinetochores, decreased kinetochore tension, and caused a mitotic block at metaphase. Therefore, dynein might deactivate spindle checkpoint after all chromosomes have bioriented at the metaphase plate (Howell et al. 2001). Putting together, it would appear that dynein plays an important role(s) not only in chromosome movement (Starr et al. 1998, Scaerou et al. 1999, Savoian et al. 2000, Sharp et al. 2000) and spindle assembly (Vaisberg et al. 1993, Echeverri et al. 1996, Gaglio et al. 1996, 1997), but also in mitotic spindle checkpoint (Howell et al. 2001).

It has been suggested that in mitosis, both Mad1 and Mad2 lie in the same checkpoint pathway that controls anaphase onset (Chen et al. 1999). Recent studies by us and others showed that Mad1 and Mad2 are two important checkpoint proteins during mammalian meiosis (Kallio et al. 2000, Wassmann et al. 2003, Zhang et al. 2004, 2005, Homer et al. 2005, Ma et al. 2005) and they check the attachment of microtubules to kinetochores by relocating their positions between spindle poles and kinetochores. However, what is responsible for the transportation (relocation) of the checkpoint proteins is still unknown. As dynein is one of the motor proteins in the cells, we hypothesize that dynein might participate in transportation of checkpoint proteins in oocyte meiosis. Therefore, in the present study, experiments were designed to examine 1) the presence and localization of dynein in oocytes during meiosis and their relationship with the transportation (relocation) of Mad1 and Mad2 and 2) the effects of dynein inhibition on the checkpoint signal transduction.

\section{Materials and Methods \\ Oocyte collection and culture}

Animal care and handling were conducted in accordance with policies on the care and use of animals promulgated by the Ethical Committee of the Institute of Zoology, Chinese Academy of Sciences. The mice with color gene type of aabbcc were from an inbred strain of Kunming white mice, a native breed widely used in biological researches in China. Prophase stage oocytes, also called immature oocytes at the germinal vesicle (GV) stage, were collected from ovaries of 4- to 6-weekold female Kunming mice at $48 \mathrm{~h}$ after equine chorionic gonadotropin (eCG) injection. Cumulus-free or cumulus-surrounded GV oocytes were collected by puncturing the large antral follicles with a needle in M2 medium (Sigma) supplemented with $60 \mu \mathrm{g} / \mathrm{ml}$ penicillin and $50 \mu \mathrm{g} / \mathrm{ml}$ streptomycin. The cumulus cell masses 
surrounding the oocytes were removed by treatment with $300 \mathrm{IU} / \mathrm{ml}$ hyaluronidase (Sigma) and repeated pipetting. Then the cumulus-free oocytes were cultured in $\mathrm{M} 2$ medium at $37^{\circ} \mathrm{C}$ in a humidified atmosphere of $5 \% \mathrm{CO}_{2}$ in air until treatment and examination.

\section{Nocodazole treatment of oocytes}

For nocodazole treatment, $10 \mathrm{mg} / \mathrm{ml}$ nocodazole in DMSO stock (Sigma) was diluted in M2 medium to give a final concentration of $20 \mu \mathrm{g} / \mathrm{ml}$ and oocytes were incubated for $10 \mathrm{~min}$, which would destroy microtubules completely (Zhang et al. 2004, 2005). After treatment, oocytes were washed thoroughly and fixed for immunofluorescence staining. In the control, oocytes were also treated in the medium with the same concentration of DMSO before examination.

\section{Immunofluorescence staining of Mad1, Mad2, dynein, microtubules, and kinetochores}

Rabbit anti-XMad1 antibody $(0.5 \mathrm{mg} / \mathrm{ml})$ in $0.1 \% \mathrm{BSA} /$ PHEM (60 mM Pipes, $25 \mathrm{mM}$ Hepes at pH 7.2, $10 \mathrm{mM}$ EGTA, $8 \mathrm{mM} \mathrm{MgSO}_{4}$ ) prepared against bacterially expressed Xenopus Mad1 was a kind gift from Dr R H Chen (Chen et al. 1998, 1999). Rabbit anti-Mad2 antibody $(0.5 \mathrm{mg} / \mathrm{ml}$ in $0.1 \%$ BSA/PHEM, $\mathrm{pH} 7.2)$ prepared against a recombinant full-length human Mad2 protein was bought from BabCO (Berkeley Antibody Company, Berkeley, CA, USA). Mouse anti-dynein (intermediate chain) antibody (IgM) was bought from Sigma. Human ANA-centromere autoantibody (CREST) was bought from Cortex Biochem ( San Leandro, CA, USA; $0.5 \mathrm{mg} / \mathrm{ml}$ in $0.1 \%$ BSA/PHEM, $\mathrm{pH}$ 7.2). In a preliminary experiment, we used both rabbit serum and FITC-conjugated goatanti-rabbit $\operatorname{lgG}$ for the immunostaining and did not find positive Mad1 or Mad2 signal; no positive dynein signal was found when both mouse IgM (Sigma) and FITCconjugated goat-anti-mouse IgM (Sigma) were used. Therefore, we found that the anti-XMad1 antibody, antiMad2 antibody, and anti-dynein antibody can specifically bind Mad1, Mad2, and dynein in mouse oocytes respectively.

As we cannot image the oocytes using three-color staining by confocal microscopy, oocytes used in this study were double stained for Mad1 and DNA, Mad2 and DNA, dynein and DNA, dynein and microtubules, Mad1 and kinetochores, and Mad2 and kinetochores. Immunofluorescence staining was based on the procedures reported previously (Zhang et al. 2004, 2005) and the oocytes were mounted on glass slides after staining and examined with a TCS-4D laser scanning confocal microscope (Leica Microsystems, Bensheim, Germany).

For Mad1 and kinetochore co-staining, oocytes were treated in $0.5 \%$ Triton X-100/PHEM for $4-5 \mathrm{~min}$ and washed thrice in PBS with $0.05 \%$ PVP, fixed in $4 \%$
paraformaldehyde/PHEM for $20 \mathrm{~min}$ and washed thrice in PBS with $0.05 \%$ PVP. After being blocked in $1 \%$ BSA/PHEM with $100 \mathrm{mM}$ glycine at room temperature for $1 \mathrm{~h}$, the oocytes were incubated in anti-Mad1 antibody diluted $1: 100$ in $1 \%$ BSA/PHEM with $100 \mathrm{mM}$ glycine at $4{ }^{\circ} \mathrm{C}$ overnight. After four washes in PBS with $0.05 \%$ Tween 20 , the oocytes were incubated with FITC-conjugated goat-anti-rabbit IgG diluted 1:200 in $1 \%$ BSA/PHEM with $100 \mathrm{mM}$ glycine for $45 \mathrm{~min}$. After three washes in PBS with $0.05 \%$ Tween 20, the oocytes were again blocked in $1 \%$ BSA/PHEM with $100 \mathrm{mM}$ glycine at room temperature for $1 \mathrm{~h}$, then stained according to the methods mentioned above except that the primary antibody is human ANA-centromere autoantibody (CREST) diluted 1:500 in 1\% BSA/PHEM with $100 \mathrm{mM}$ glycine and the second antibody is Alexa 568conjugated goat-anti-human IgG (molecular probe) diluted $1: 200$ in 1\% BSA/PHEM with $100 \mathrm{mM}$ glycine.

For Mad2 and kinetochore co-staining, all steps were the same as those for Mad1 and kinetochore co-staining except that for the first round of immunoreaction; the primary antibody is rabbit anti-Mad2 antibody diluted $1: 100$ in $1 \%$ BSA/PHEM with $100 \mathrm{mM}$ glycine and the second antibody is FITC-conjugated goat-anti-rabbit $\lg \mathrm{G}$ diluted 1:200 in 1\% BSA/PHEM with $100 \mathrm{mM}$ glycine.

For Mad1 and DNA staining, all steps were the same as those for Mad1 and kinetochore co-staining except that after the first round of immunoreaction, the oocytes were stained with $10 \mathrm{mM}$ propidium iodide in PBS with $0.05 \%$ Tween 20 for 2-3 min before examination.

For Mad2 and DNA or dynein and DNA staining, all steps were the same as those for Mad1 and DNA staining except that the primary antibody is rabbit anti-Mad2 antibody diluted 1:100 in 1\% BSA/PHEM with $100 \mathrm{mM}$ glycine and the second antibody is FITC-conjugated goat-anti-rabbit IgG diluted $1: 200$ in 1\% BSA/PHEM with $100 \mathrm{mM}$ glycine, or that the primary antibody is mouse anti-dynein antibody (IgM) diluted 1:100 in 1\% BSA/PHEM with $100 \mathrm{mM}$ glycine and the second antibody is FITC-conjugated goat-anti-mouse IgM diluted 1:200 in 1\% BSA/PHEM with $100 \mathrm{mM}$ glycine.

For dynein and microtubules co-staining, all steps were the same as those for Mad1 and nuclei staining except that the primary antibody was mixture of mouse anti-dynein antibody (IgM) diluted 1:100 in 1\% BSA/PHEM with $100 \mathrm{mM}$ glycine and mouse anti- $\alpha$-tubulin antibody $1: 16000$ in $1 \%$ BSA/PHEM with $100 \mathrm{mM}$ glycine, and the second antibody was mixture of FITC-conjugated goatanti-mouse IgM diluted 1:200 in 1\% BSA/PHEM with $100 \mathrm{mM}$ glycine and FITC-conjugated goat-anti-mouse $\lg 1: 200$ in 1\% BSA/PHEM with $100 \mathrm{mM}$ glycine.

\section{Microinjection of anti-dynein antibody into immature oocytes}

To study the effects of dynein on the nuclear maturation and the localization of Mad1 or Mad2, anti-dynein 
antibody $(0.5 \mathrm{mg} / \mathrm{ml}$ in $0.1 \%$ BSA/PHEM, pH 7.2) was injected into the cytoplasm of fully grown oocytes at GV stage as previously reported (Shannon et al. 2002, Tsurumi et al. 2004). Isobutylmethylxanthine was added to $\mathrm{M} 2$ medium to produce a final concentration of $0.2 \mu \mathrm{M}$ to prevent GV breakdown during injection and treatment. A microinjection volume of $7 \mathrm{pl}$ per oocyte was used in all experiments. The oocytes were washed and cultured in M2 medium for 8 (to M-I stage) or $16 \mathrm{~h}$ (to $\mathrm{M}$-II stage) before confocal examination of Mad1 and DNA or Mad2 and DNA. Subgroups were cultured for $9.5,12,14$, or $16 \mathrm{~h}$ to examine nuclear maturation. Mouse IgM or culture medium was also injected into other oocytes to act as controls.

\section{Statistical analysis}

All experiments were repeated thrice. All percentage data were subjected to arc sine transformation before significance analysis. Data were analyzed by ANOVA of EXCEL and multiple range tests were conducted with $q$-test. Differences at $P<0.05$ were considered significant.

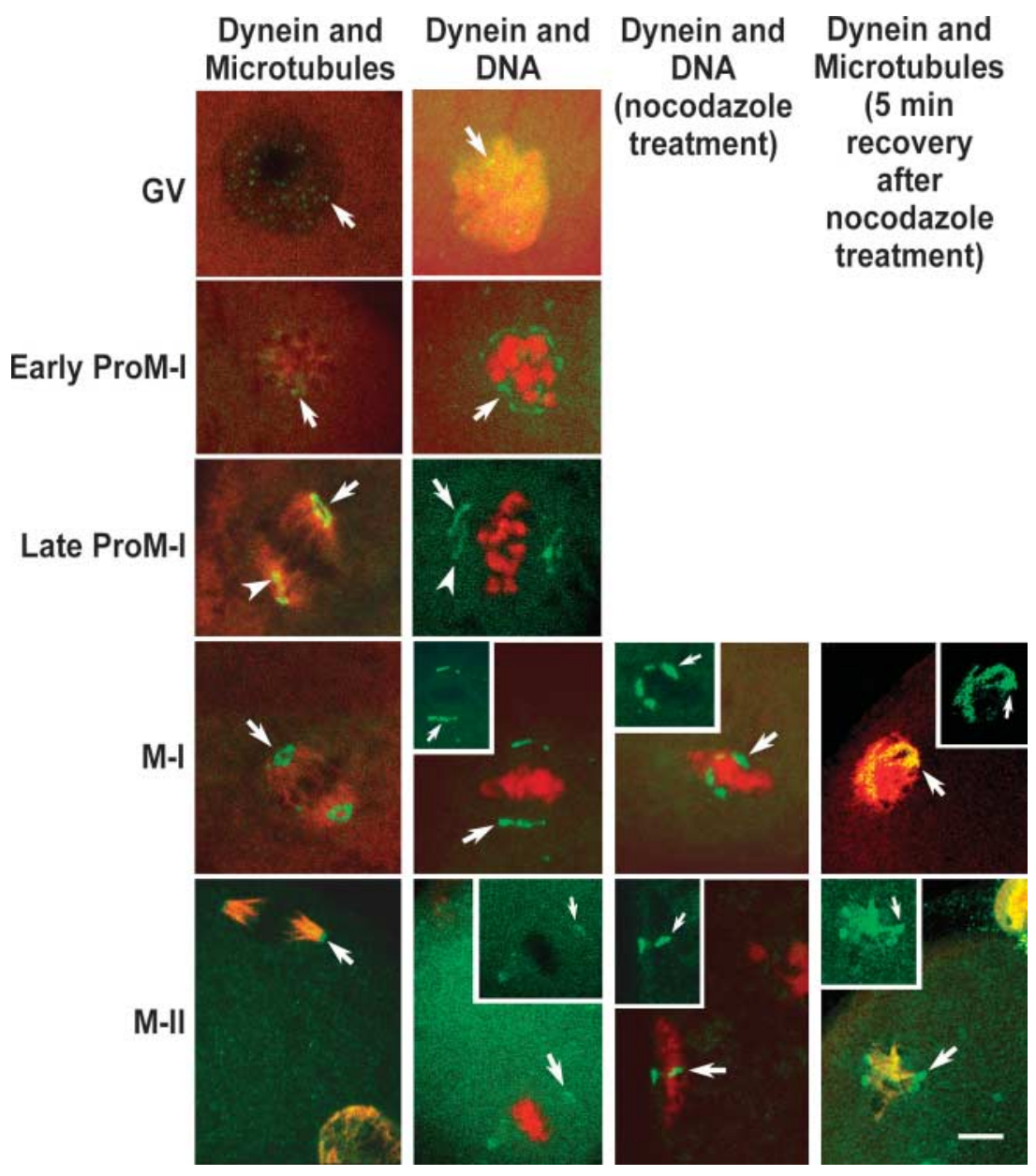

\section{Results}

\section{Localization of dynein in normal or nocodazole-treated oocytes}

As shown in Fig. 1, when oocytes were at GV and early ProM-I stages, dynein was mainly distributed inside or around the nucleus and chromosomes. When the oocytes were at late ProM-I stage, most dynein aligned well at spindle poles (Fig. 1, arrow), while part of dynein stranded off spindle poles (Fig. 1, arrow head). When the oocytes reached $M-I$ and $M-I I$ stages, dynein was assembled at the spindle poles and no dynein signals were observed around the chromosomes. When the oocytes were treated with $20 \mu \mathrm{g} / \mathrm{ml}$ nocodazole to depolymerize the spindles, in $81.2 \%(82 / 101)$ of $\mathrm{M}-\mathrm{I}$ and $73.6 \%$ (57/77) of M-II oocytes, all dynein moved from the spindle poles to the region of chromosomes, but no dynein signals were observed among the chromosomes. Since co-staining of dynein and microtubules right after nocodazole treatment was inaccessible due to great elimination of microtubules, oocytes were recovered in $\mathrm{M} 2$ medium for $5 \mathrm{~min}$ followed by the staining procedure above. It was found that dynein
Figure 1 Confocal microscopic images of microtubules, nuclei, and dynein localization in normal (left two columns) and nocodazoletreated (right two column) oocytes. Red images represent microtubules (from left, columns 1 and 4) or nucleus (from left columns 2 and 3) and green images represent dynein. Arrows indicate dynein. GV, germinal vesicle; ProM-I, prometaphase I; M-I, metaphase I; M-II, metaphase II; Pb, polar body. Bar $=10 \mu \mathrm{m}$. 
localized around microtubule growing from the Microtuble-organising centre (MTOC) at chromosomes.

\section{Localization of Mad1 and Mad2 in normal or nocodazole-treated oocytes}

Since Mad1 and Mad2 localization at different meiotic stages were reported before (Zhang et al. 2004, 2005), in this study, we focused on the co-staining of these proteins and kinetochores of the oocytes at prometaphase and metaphase (I and II) stages. Kinetochores and Mads were clearly labeled at these stages. We found that both Mad1 and Mad2 had two existing states during meiosis: one existed in the cytoplasm (did not bind to kinetochores) and was named as cytoplasmic Mad1 or cytoplasmic Mad2, while the other bound to kinetochores and was named as kinetochore Mad1 or kinetochore Mad2. Since cytoplasmic Mads at M-I or M-II stage were mainly at spindle poles, we specifically called $\mathrm{M}$-I or M-II cytoplasmic Mads as spindle pole Mads. The detailed localization and equilibrium between two states of proteins are as follows:

As shown in Fig. 2, most Mad1 in the oocytes at ProM-I stage existed in an assembled status around the chromosomes and some were individually distributed between the chromosomes. Part of individual Mad1 bound to kinetochores and the remaining existed as cytoplasmic Mad1. When the oocytes reached M-I and M-II stages, Mad1 staining was mainly found at the spindle poles in an assembled status, only very little Mad1 bound to kinetochores.
As shown in Fig. 2, Mad2 also existed in assembled and individual states in the oocytes at ProM-I stage. However, all Mad2 was scattered within the chromosomes; co-staining of Mad2 and kinetochores indicated that most individual Mad2 was kinetochore Mad2 that completely bound to kinetochores, while the assembled Mad2 was cytoplasmic Mad2 that did not bind to kinetochores. When the oocytes reached $\mathrm{M}-\mathrm{I}$ and $\mathrm{M}-\mathrm{II}$ stages, cytoplasmic and kinetochore Mad2 were completely separated: cytoplasmic Mad2 moved to the spindle poles in an assembling status, a few part of Mad2 bound to kinetochores, however, kinetochore Mad2 signal at $\mathrm{M}-\mathrm{I}$ or $\mathrm{M}$-II stage seemed stronger than kinetochore Mad1 signal.

In order to examine Mad1 and Mad2 changes in the oocytes after microtubules and/or spindles were disassembled, we used $20 \mu \mathrm{g} / \mathrm{ml}$ nocodazole to treat the oocytes. In a preliminary experiment, we found that all microtubules were destroyed when this concentration of nocodazole was used and spindles could reassemble after the removal of nocodazole in culture.

As shown in Fig. 2, nocodazole-treatment of ProM-I oocytes did not change the status of cytoplasmic and kinetochore Mad1 when compared with control oocytes and most was cytoplasmic Mad1. When the oocytes at M-I and M-II stages were treated with nocodazole, most cytoplasmic Mad1 moved from the spindle poles to the chromosomes in an assembled status, but some individual Mad1 was found scattered among the chromosomes and bound to kinetochores as indicated by co-staining of Mad1 and kinetochores and in contrast,

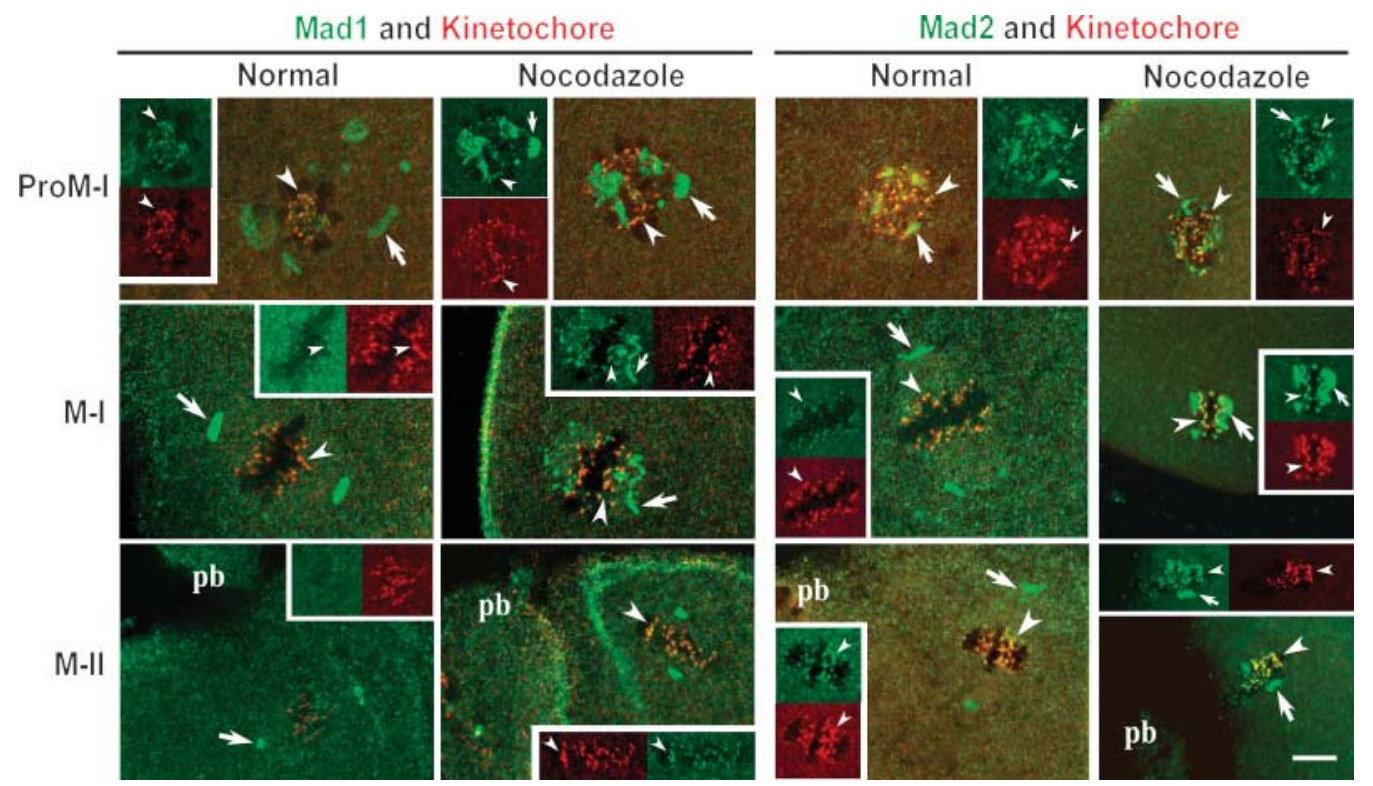

Figure 2 Confocal microscopic images of co-staining of Mad1 and kinetochores or Mad2 and kinetochores in normal or nocodazole-treated oocytes during meiosis. Each panel contains two single-channel insets with equal magnification that showed Mad or kinetochore staining. Arrows indicate cytoplasmic Mad, and arrowheads indicate kinetochore Mad. Red images represent kinetochores, green images represent Mad, and yellow images represent overlay of kinetochores and Mad. ProM-I, prometaphase I; M-I, metaphase I; M-II, metaphase II; Pb, polar body. Bar=10 $\mu$ m. 
kinetochore Mad1 signals in nocodazole-treated oocytes seemed much stronger than in normal oocytes.

The localization of Mad2 in oocytes at ProM-I stage treated with nocodazole was similar to those in the oocytes without nocodazole treatment. However, in $85.4 \%(111 / 130)$ of $\mathrm{M}-\mathrm{I}$ and $78.1 \%(68 / 87)$ of $\mathrm{M}-\mathrm{II}$ oocytes, localization of Mad2 changed after nocodazole treatment: all cytoplasmic Mad2 moved from the spindle poles to the chromosomes but did not bind to kinetochores. Co-staining of Mad2 and kinetochores indicated that all Mad2 that scattered in the chromosomes were kinetochore Mad2, which seemed stronger than in normal oocytes. And same as in normal M-I or M-I oocytes, kinetochore Mad2 signal seemed stronger than Mad1 signal in nocodazole-treated oocytes.

\section{Effects of anti-dynein antibody on meiotic progress in the oocytes}

As shown in Fig. 3A, at $9.5 \mathrm{~h}$ after injection, in control oocytes about $52.3 \%(57 / 109)$ were at M-I stage and $27.5 \%(30 / 109)$ were at A-I-T-I stage, while in antidynein antibody-injected oocytes, significantly more $(76.1 \%, 71 / 93)$ oocytes were at $\mathrm{M}$ - I stage and significantly less oocytes $(5.4 \%, 5 / 93)$ were at A-I-T-I stage. As shown in Fig. 3B, at 12, 14, and $16 \mathrm{~h}$ after injection, about $75.6 \%(113 / 150), 81.8 \%(122 / 150)$, and $85.5 \%$ (128/150) of control oocytes released the first polar body, while only $41.5 \%$ (62/150), $60.7 \%$ (91/150), and $75.3 \%$ $(112 / 150)$ of anti-dynein antibody-injected oocytes released the first polar body, which were significantly $(P<0.05)$ lower than in control. The oocytes that did not release the first polar body were at ProM-I or M-I stage. However, we did not find the difference in the morphology of polar body and meiotic spindle.

\section{Effect of anti-dynein antibody on the localization of Mad1 and Mad2 in the oocytes}

In order to examine the relationship between dynein and checkpoint proteins (Mad1 and Mad2), anti-dynein antibody was injected into the oocytes at the GV stage, and then the injected oocytes were cultured for examination of the localization of Mad1 and Mad2 after the oocytes reached $\mathrm{M}-\mathrm{I}, \mathrm{A}-\mathrm{I}$, and $\mathrm{M}-\mathrm{II}$ stages.

As shown in Fig. 4A-C, it was found that $64.6 \%$ (82 of 127 ) of M-I, $75 \%$ (12 of 16 ) of A-I and $60.2 \%$ (53 of 88 ) of M-II oocytes had abnormal Mad1 localization, in which most cytoplasmic Mad1 was no longer localized at the spindle poles, but irregularly localized around the chromosomes. In addition, all cytoplasmic Mad1 still existed in an assembling status and there were very few kinetochore Mad1; while in the control, Mad1 in most oocytes were at the spindle poles regardless of the oocyte stages. However, after nocodazole treatment in antibody-injected oocytes, as in control oocytes, cytoplasmic Mad1 still recruited to chromosomes and
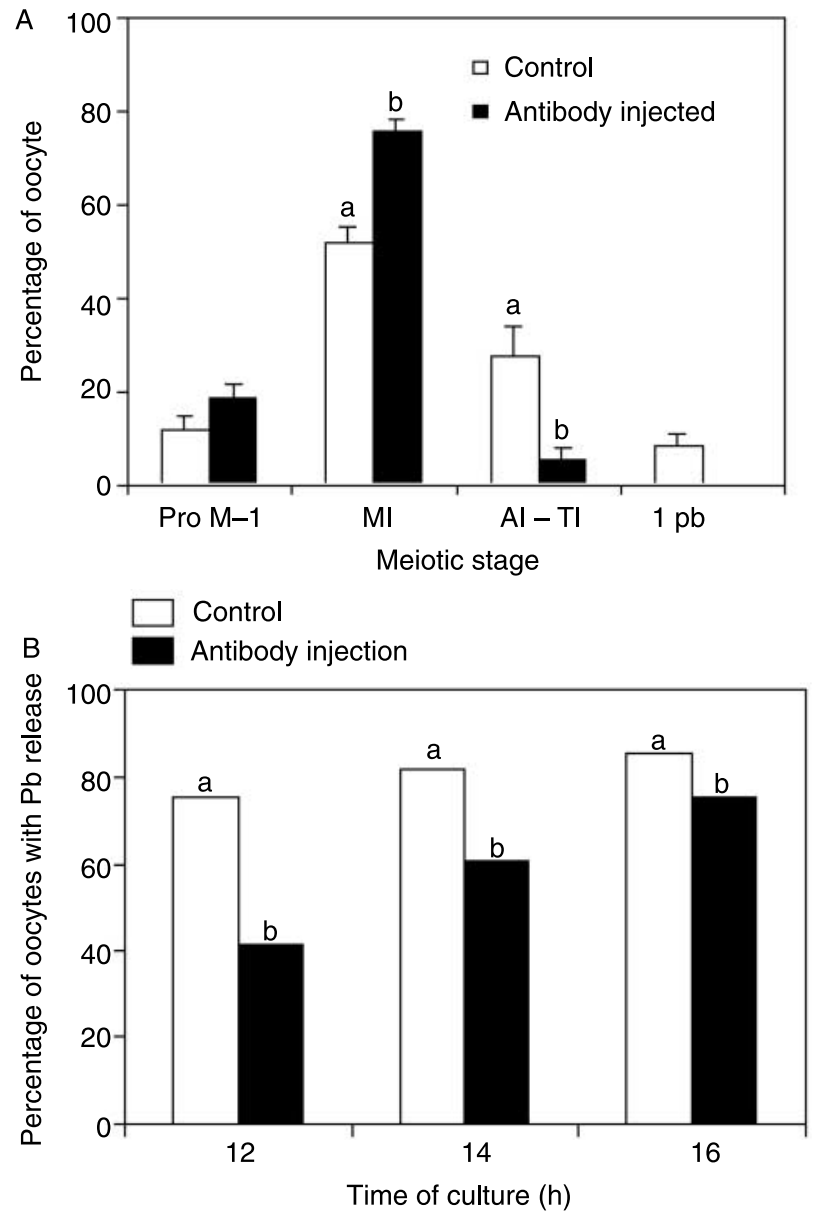

Figure 3 (A) Nuclear progression of mouse oocytes after anti-dynein antibody injection at the GV stages. Oocytes were examined after cultured for $9.5 \mathrm{~h}$. ProM-I, prometaphase I; M-I, metaphase I; A-I-T-I, anaphase I to telophase I; $1 \mathrm{~Pb}$, first polar body. ${ }^{\mathrm{a}, \mathrm{b}} P<0.05$. (B) Nuclear progression of mouse oocytes after anti-dynein antibody injection at the GV stages. Oocytes were examined after cultured for 12,14 , or $16 \mathrm{~h}$. ${ }^{\mathrm{a}, \mathrm{b}} \mathrm{P}<0.05$

kinetochore Mad1 staining still increased (Fig. 4A and B). Co-staining in proM-I or M-I oocytes further support that Mad1 scattering in the chromosomes was kinetochore Mad1 (Fig. 4B).

As shown in Fig. $5 \mathrm{~A}-\mathrm{C}$, at 8 or $16 \mathrm{~h}$ after anti-dynein antibody injection, it was found that significantly $(P<0.05)$ more injected oocytes had abnormal Mad2 localization than that in control. In $67.2 \%(80 / 119)$ of M-I and $61.3 \%(76 / 124)$ of M-II oocytes, most cytoplasmic Mad2 was not localized at the spindle poles but between the spindle poles and the chromosomes, some were even closer to the chromosomes. A little Mad2 was scattered in the chromosomes. In $66.7 \%(12 / 18)$ of A-I oocytes, Mad2 was assembled at the spindle midzone, but no Mad2 was found in the chromosomes. While in the control oocytes at M-I and M-II stages, most Mad2 was at the spindle poles and no Mad2 was observed in the control A-I oocytes. However, after nocodazole treatment in antibody-injected 
A

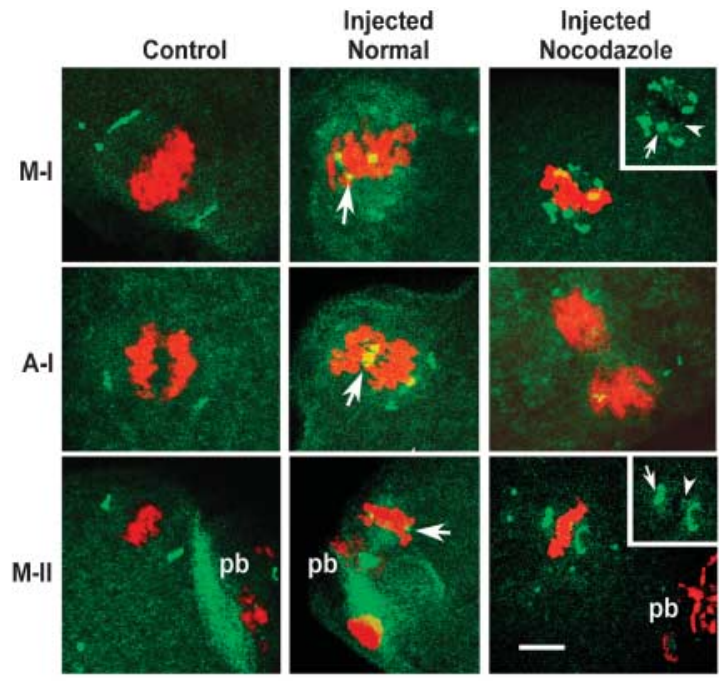

\section{B Anti-dynein antibody injected Mad1 and Kinetochore}
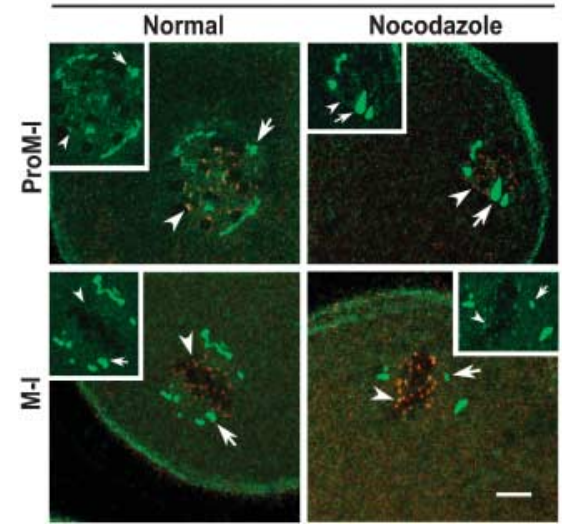

C

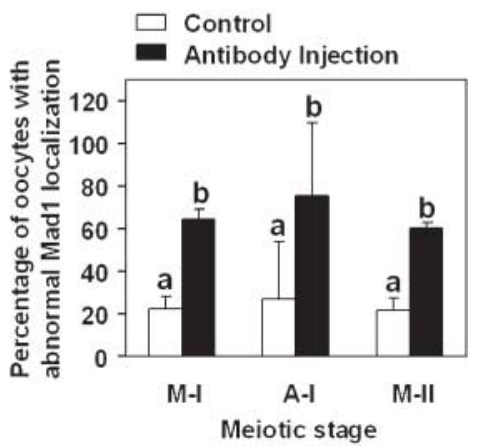

Figure 4 (A) Confocal microscopic images of Mad1 localization in normal or nocodazoletreated anti-dynein antibody-injected oocytes. Oocytes at the GV stage were injected with anti-dynein antibody and cultured for 8-16 h before examination. Control: no antibody injection. Red images represent nucleus and green images Mad1; some panels contain singlechannel insets of Mad1 with equal magnification. Arrows indicate abnormal cytoplasmic Mad1 localization in normal injected oocytes or relocated Mad1 in nocodazole-treated oocytes, arrow heads indicate kinetochore Mad1. $\mathrm{Pb}$, polar body. Bar $=10 \mu \mathrm{m}$. (B) Confocal microscopic images of co-staining of Mad1 and kinetochores in normal or nocodazole-treated anti-dynein antibody-injected oocytes at ProM-I or M-I stage. Each panel contains single-channel inset of Mad1 with equal magnification. Arrows indicate cytoplasmic Mad1 and arrowheads kinetochore Mad1. Red images represent kinetochores, green images represent Mad, and yellow images represent overlay of kinetochores and Mad1. ProM-I, prometaphase I; M-I, metaphase I. (C) Proportions of oocytes with abnormal Mad1 localization between anti-dynein antibody-injected and control oocytes at M-I, A-I, and M-II stages. ${ }^{\mathrm{a}, \mathrm{b}} \mathrm{P}<0.05$. oocytes, as in control oocytes, cytoplasmic Mad2 still recruit to chromosomes and kinetochore Mad2 staining still increased (Fig. 5A and B). Co-staining of Mad2 and kinetochores in proM-I or M-I oocytes further support that Mad2 scattering in the chromosomes was kinetochore Mad2 (Fig. 5B).

\section{Discussion}

In the present study, same as previous studies in mitosis (Chen et al. 1998, 1999) and meiosis (Zhang et al. 2004, 2005), we found that two different states of Mad1 and Mad2 were present in the mouse oocytes during meiosis: cytoplasmic Mad and kinetochore Mad. These two parts were in a movable equilibrium in accordance with the relationship between microtubules and chromosomes. In the ProM-I oocytes, in which the connection between microtubules and chromosomes had not established and a bi-polar spindle had not formed, a larger percentage of Mads were kinetochore-associated this was also a pool of cytoplasmic Mads around the chromosomes. When the oocytes reached $\mathrm{M}-\mathrm{I}$ or $\mathrm{M}$-II stages in which a complete spindle had formed and chromosomes had aligned at the equator of the spindle, kinetochore Mads were reduced and cytoplasmic Mads were increased, and most Mads moved away from the chromosomes (depleted from kinetochores) and were located at the spindle poles. When the microtubules/spindles in the oocytes at $\mathrm{M}-\mathrm{I}$ and $\mathrm{M}$-II stages were destroyed by nocodazole treatment, spindle pole Mads recruited to the chromosomes and a part of them rebound to kinetochores. We used co-staining of Mad and kinetochores to verify these results in the present study. It is clear that the equilibrium shift and location changes of Mads are related to the connection between microtubules and chromosomes.

Kinetochore Mad is the most important Mad that plays a critical role in controlling anaphase onset (Chen et al. 1998, 1999). However, what are the functions of cytoplasmic Mad? From the dynamic changes of Mads during cell cycles, it would appear that cytoplasm maintains a Mads pool around the nucleus and there is a 'crosstalk' between cytoplasmic and kinetochore Mads. Before M-I stage, since cytoplasmic Mads stand 
A

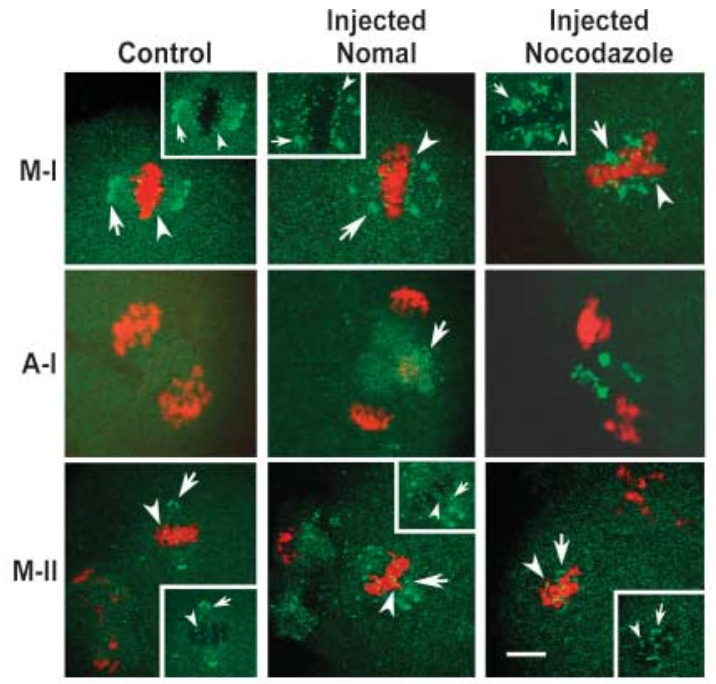

B
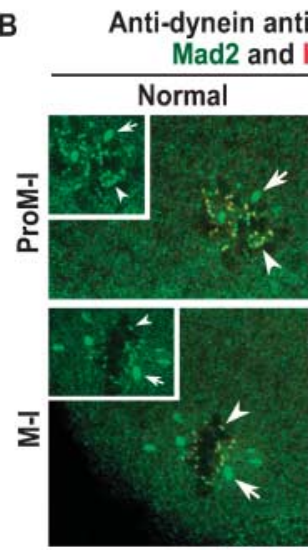

ody injected
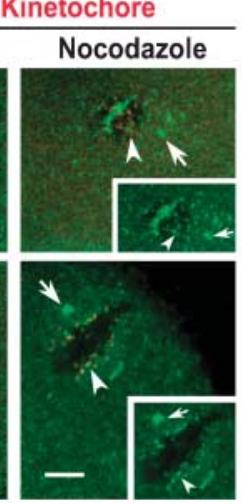

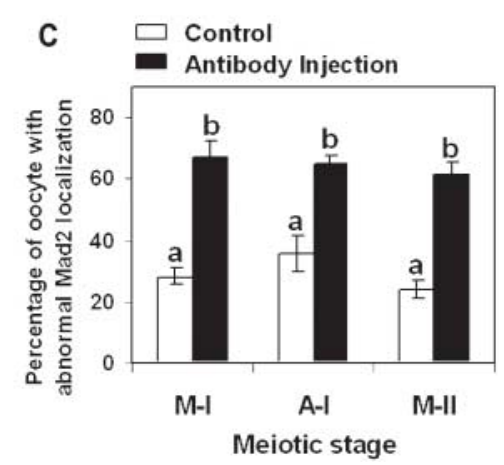

Figure 5 (A) Confocal microscopic images of Mad2 localization in anti-dynein antibodyinjected oocytes. Oocytes at the GV stage were injected with anti-dynein antibody and cultured for $8-16 \mathrm{~h}$ before examination. Control: no antibody injection. Red images represent nucleus and green represent Mad2; some panels contain single-channel insets of Mad2 with equal magnification. Arrows indicate abnormal cytoplasmic Mad1 localization in normal injected oocytes or relocated cytoplasmic Mad2 in nocodazole-treated oocytes, arrow heads indicate kinetochore Mad2. $\mathrm{Pb}$, polar body. Bar $=10 \mu \mathrm{m}$. (B) Confocal microscopic images of co-staining of Mad1 and kinetochores in normal or nocodazole-treated anti-dynein antibodyinjected oocytes at ProM-I or M-I stages. Each panel contains single-channel inset of Mad2 with equal magnification. Arrows indicate cytoplasmic Mad2 and arrowheads indicate kinetochore Mad2. Red images represent kinetochores, green images represent Mad2, and yellow images represent overlay of kinetochores and Mad2. ProM-I, prometaphase I; M-I, metaphase I. (C) Proportions of oocytes with abnormal Mad2 localization between anti-dynein antibodyinjected and control oocytes at M-I, A-I, and M-II stages. ${ }^{\mathrm{a}, \mathrm{b}} \mathrm{P}<0.05$. close to kinetochore Mads, the turnover between these two states is quick and strong which keeps the checkpoint active. When oocytes progress into M-I stage, cytoplasmic Mads move far away from kinetochores Mads, so the turnover has been greatly slowed down, which inactivate the checkpoint. When the connection between microtubules and chromosomes is destroyed, the spindle pole Mads are transported back to the chromosomes and again come close to kinetochore Mads, which recovers the normal turnover between cytoplasmic and kinetochore Mads and, in turn, activate the checkpoint. The oocytes always keep a pool of extra Mads in the cytoplasm, which may explain why there are always cytoplasmic Mads in the oocytes. These results were similar to those observed in other cells during mitosis (Chen et al. 1998, 1999).

Recently, Luo et al. (2004) found that Mad2 had two folding states, named N1-Mad2 and N2-Mad2, they were in a movable equilibrium, and that N2-Mad2 plays more important roles in the inhibition of anaphase onset. Overexpression of Mad2 mutant that specially insulated the function of N2-Mad2 partially blocked checkpoint pathway (Luo et al. 2004). Our data suggest that Mad1 and Mad2, as key components of meiotic checkpoint, might also have two different states in a movable equilibrium and the part at kinetochores might play more important roles in metaphase arrest. However, further studies are necessary to examine whether there is structural differences between kinetochore and cytoplasmic Mads. The relationship between Mad1 and Mad2 is still unknown. Luo et al. (2004) found that Mad2's two folding states could transform into each other and this transformation could be promoted by Mad1. It has also been found that in mitosis, some Mad2 bound to Mad1 and other Mad2 was free from Mad1, and a tight complex of Mad1 and Mad2 is important for a functional checkpoint (Chen et al. 1999). In addition, Mad1 recruits Mad2 to unattached kinetochores (Chen et al. 1998). However, the relationship between Mad1 and Mad2 is still unclear in meiosis.

It has been reported that transportation is one of dynein's most important functions (Gibbons \& Rowe 1965, Lye et al. 1987, Paschal \& Vallee 1987, Howell et al. 2001). Among the transient motor proteins at the kinetochores, centrosome protein (CENP) and mitotic centromere associated kinesin (MCAK)/Xenopus kinesin 
catastrophe modulator 1 (XKCM1) are plus-oriented motors (from spindle poles to kinetochores), while dynein is a minus-oriented motor (from kinetochores to spindle poles; Barton \& Goldstein 1996, Maney et al. 1999, Hunter \& Wordeman 2000). In the present study, we suggest that dynein plays an important role(s) in spindle checkpoint inactivation and this function is related to two key checkpoint proteins: Mad1 and Mad2. The functions of dynein and its connection with Mads are manifested as follows:

First, dynein participate in checkpoint inactivation. We checked whether anti-dynein antibody injection will affect oocytes meiotic progression. We found that significantly more oocytes were still at M-I stage than control after 9.5-h culture, and significantly less oocytes released first polar body after 16-h culture, which means oocytes had been delayed entering anaphase. This is a reverse phenomena compared with that after inhibition of two key checkpoints Mad1 and Mad2, which caused premature anaphase (Zhang et al. 2004, 2005). Therefore, we inferred that instead of happened on time, the checkpoint inactivation had been delayed after antidynein antibody injection.

Second, dynein' function in checkpoint inactivation is closely related to Mads' relocation from prometaphase to metaphase. As suggested above, Mads' relocation from prometaphase to metaphase is important for checkpoint inactivation. When dynein was inhibited by antibody injection, the relocation had been greatly blocked: only a small part of Mads localized at spindle poles, a large part localized between spindle poles and chromosomes, some stranded as close to chromosomes as after nocodazole treatment. Since dynein inhibition delayed checkpoint inactivation, it is reasonable that this delay was due to the relocation of Mads. How could this have happened? It could be that when the cytoplasmic Mads stand near the chromosomes, the equilibrium between cytoplasmic Mads and kinetochore Mads had been modified similar to that at ProM-I, which activated the checkpoint again.

Third, dynein inactivate checkpoint through transporting cytoplasmic Mads, not kinetochore Mads. This can be inferred from the following: first, after dynein inhibition, Mads still co-localized with kinetochores in normal or nocodazole-treated oocytes before M-I (Figs 4 and 5). These results indicate that Mad1 and Mad2 binding to kinetochore were not affected by anti-dynein antibody injection. If dynein was responsible for Mad1 and Mad2 binding to kinetochore, anti-dynein injection would prevent Mad1 and Mad2 binding to kinetochore, thus oocytes would enter an early anaphase and show abnormal polar body release as Mad2 inhibition observed by anti-Mad2 antibody injection experiment (Gorbsky et al. 1998, Zhang et al. 2004). However, we did not see similar results as those by Mad2 inhibition, but reverse results. These results indicate that Mad1 and Mad2 binding to kinetochores is dynein-independent before M-I stage. Second, Most dynein localized around chromosomes, but did not bind to kinetochore during prometaphase (Fig. 1) and after microtubule destruction, dynein still localized around chromosomes instead of on kinetochore. Hence, it is impossible for dynein to move onto kinetochores and detach Mads away from kinetochore. Third, considering Mads still localized at kinetochore during metaphase, it seemed not necessary that Mads are completely detached from kinetochore to inactivate checkpoint during metaphase. The equilibrium change between cytoplasmic and kinetochore Mads may be more important for checkpoint switch, while dynein inhibition changed the equilibrium by relocating cytoplasmic Mads.

Finally, although dynein inhibition prevents Mad transportation during the meiosis, microtubule formation, spindle structure, and chromosome alignment were not affected. This could be due to the following reasons: first, as shown in Fig. 1, at GV stage, dynein mainly localized inside or around nucleus, while we injected the anti-dynein antibody into cytoplasm (quick death happened if we directly injected antibody into nucleus), so there is a gradient effect, which is normal in any antibody-injection experiment and it is possible that dynein inside the nucleus was only partially blocked since it is away from the injection site. Second, also from Fig. 1, dynein expression level seemed to increase as oocytes develop from GV to $\mathrm{M}-\mathrm{I}$, since we inject antibody at GV stage, we might just partially knocked down dynein's activity. Certainly it is not surprising that, in partial knockdown oocytes, spindle morphology still kept intact since there is still dynein functioning there. And from the Mads localization after knockdown (Figs 4A, B and 5A, B), we can find that there are still some Mads near or at the poles, which may suggest that dynein still partially functioned. Nevertheless, partial knockdown already caused significant effect on oocytes development even though the spindles are kept intact, which in reverse indicate dynein's importance. Since partial dynein inhibition had not affected spindle structure, there is little possibility that the relocation of Mads after dynein inhibition was due to change of spindle structure as reported before (Zhang et al. 2005). So in this case, partial knockdown may be more preferable for ascertaining the real cause of Mads relocation than complete knockdown. Third, chromosome movement and microtubule formation may be not sensitive to dynein inhibition, which were reported in mitosis (Gaglio et al. 1997, Howell et al. 2001), hence though our results could not certify that dynein directly relocate Mads, it suggests that dynein participates in cytoplasmic Mads relocation, specifically transporting cytoplasmic Mads from chromosomes to spindle poles through its minus-end motor activity. Inhibition of dynein prevented Mads transportation from kinetochore to spindle poles which was also observed in mitosis. In Ptkl cells, microinjection of purified p50 dynamitin 
protein or anti-dynein antibody also prevented Mad2, BubR1, CENP-E, and 3F3/2 phosphoantigen from transporting to the spindle poles and prevented Mad2 detachment from the kinetochores (Howell et al. 2001). Therefore, it would be possible that dynein's function as a checkpoint protein transportation is the same between mitosis and meiosis.

In conclusion, the present study indicates that as seen in mitosis, dynein may participate in spindle checkpoint inactivation by transporting cytoplasmic Mads from chromosomes to spindle poles. The equilibrium between cytoplasmic and kinetochore Mads is essential for checkpoint activation/inactivation. Inhibition of dynein causes cytoplasmic Mads to stay near chromosomes instead of being transported to poles during $\mathrm{M}-\mathrm{I}$, thus kept the equilibrium between cytoplasmic and kinetochore Mads similar to proM-I, which in turn delays checkpoint inactivation. These results indicate that dynein is important for checkpoint inactivation during oocyte meiosis.

\section{Acknowledgements}

We thank Dr R H Chen for kindly providing anti-Mad1 antibody, Dr G J Gorbsky for providing information for us to obtain the CREST antibody, Prof. D Y Chen for providing microinjection facilities, Mrs S W Li for her assistance in confocal microscopy and $\mathrm{Y} J \mathrm{~W}$ Wang for reading the manuscript. The authors declare that there is no conflict of interest that would prejudice the impartiality of this scientific work.

\section{Funding}

National Natural Science Foundation of China (No 30570944, 30430530, and 30225010), National Basic research Program (2006CB504004 and 2006CBOF1001), and CAS Knowledge Innovation Program (KSCX2-YW-R-52).

\section{References}

Barton NR \& Goldstein LS 1996 Going mobile: microtubule motors and chromosome segregation. PNAS 93 1735-1742.

Brunet S, Pahlavan G, Taylor S \& Maro B 2003 Functionality of the spindle checkpoint during the first meiotic division of mammalian oocytes. Reproduction 126 443-450.

Chen RH, Shevchenko A, Mann M \& Murray AW 1998 Spindle checkpoint protein Xmad1 recruits Xmad2 to unattached kinetochores. Journal of Cell Biology 143 283-295.

Chen RH, Brady DM, Smith D, Murray AW \& Hardwick KG 1999 The spindle checkpoint of budding yeast depends on a tight complex between the Mad1 and Mad2 proteins. Molecular Biology of the Cell 10 2607-2618.

Escheverri CJ, Paschal BM, Vaughan KT \& Vallee RB 1996 Molecular characterization of the 50-kD subunit of dynactin reveals function for the complex in chromosome alignment and spindle organization during mitosis. Journal of Cell Biology 132 617-633.

Gaglio T, Saredi A, Bingham JB, Hasbani MJ, Gill SR, Schroer TA \& Compton DA 1996 Opposing motor activities are required for the organization of the mammalian mitotic spindle pole. Journal of Cell Biology 135 399-414.
Gaglio T, Dionne MA \& Compton DA 1997 Mitotic spindle poles are organized by structural and motor proteins in addition to centrosomes. Journal of Cell Biology 138 1055-1066.

Gibbons IR \& Rowe A 1965 Dynein: a protein with adenosinetriphosphatase activity from cilia. Science 149423.

Gorbsky GJ, Chen RH \& Murray AW 1998 Microinjection, of antibody to Mad2 protein into mammalian cells in mitosis induces premature anaphase. Journal of Cell Biology 141 1193-1205.

Homer HA, McDougall A, Levasseur M, Yallop K, Murdoch A \& Herbert M 2005 Mad2 prevents aneuploidy and premature proteolysis of cyclin B and securing during meiosis I in mouse oocytes. Genes \& Development 19 202-207.

Howell BJ, McEwen BF, Canman JC, Hoffman DB, Farrar EM, Rieder CL \& Salmon ED 2001 Cytoplasmic dynein/dynactin drives kinetochore protein transport to the spindle poles and has a role in mitotic spindle checkpoint inactivation. Journal of Cell Biology 155 1159-1172.

Hoyt MA 2001 A new view of the spindle checkpoint. Journal of Cell Biology 154 909-911.

Hoyt MA, Totis L \& Roberts BT 1991 S. cerevisian genes required for cell cycle arrest in response to loss of microtubule function. Cell $\mathbf{6 6}$ 507-517.

Hunter AW \& Wordeman L 2000 How motor proteins influence microtubule polymerization dynamics. Journal of Cell Science $\mathbf{2 4}$ 4379-4389.

Kallio M, Eriksson JE \& Gorbsky GJ 2000 Differences in spindle association of the mitotic checkpoint protein Mad2 in mammalian spermatogenesis and oogenesis. Developmental Biology 225 112-123.

King JM, Hays TS \& Nicklas RB 2000 Dynein is a transient kinetochore component whose binding is regulated by microtubule attachment, not tension. Journal of Cell Biology 151 739-748.

Li R \& Murray AW 1991 Feedback control of mitosis in budding yeast. Cell 66 519-531.

Luo X, Tang Z, Xia G, Wassmann K, Matsumoto T, Rizo J \& Yu H 2004 The Mad2 spindle checkpoint protein has two distinct natively folded states. Nature Structural \& Molecular Biology 11 338-345.

Lye RJ, Porter ME, Scholey JM \& McIntosh JR 1987 Identification of a microtubule-based cytoplasmic motor in the nematode $C$. elegans. Cell 51 309-318.

Ma W, Zhang D, Hou Y, Li YH, Sun QY, Sun XF \& Wang WH 2005 Reduced expression of Mad2, Bcl-2 and MAP kinase activity in pig oocytes after in vitro aging are associated with defects of sister chromatid segregation during meiosis II and embryo fragmentation after activation. Biology of Reproduction 72 373-383.

Maney T, Ginkel LM, Hunter AW \& Wordeman L 1999 The kinetochore of higher eucaryotes: a molecular view. International review of cytology 194 67-132.

Paschal BM \& Vallee RB 1987 Retrograde transport by the microtubule associated protein MAP-1C. Nature 330 181-183.

Pfarr CM, Coue M, Grissom PM, Hays TS, Porter ME \& McIntosh JR 1990 Cytoplasmic dynein is localized to kinetochores during mitosis. Nature 345 263-265.

Rieder CL \& Salmon ED 1998 The vertebrate cell kinetochore and its roles during mitosis. Trends in Cell Biology 8 310-318.

Roberts BT, Farr KA \& Hoyt MA 1994 The Saccharomyces cerevisian checkpoint gene $B U B 1$ encodes a novel protein kinase. Molecular Biology of the Cell 14 8282-8291.

Savoian MS, Goldberg ML \& Rieder CL 2000 The rate of chromosome poleward motion is attenuated in Drosophila zw10 and rod mutants. Nature Cell Biology 2 948-952.

Scaerou F, Aguilera I, Saunders R, Kane N, Blottiere L \& Karess R 1999 The rough deal protein is a new kinetochore component required for accurate chromosome segregation in Drosophila. Journal of Cell Science 112 3757-3768.

Shannon KB, Canman JC \& Salmon ED 2002 Mad2 and BubR1 function in a single checkpoint pathway that responds to a loss of tension. Molecular Biology of the Cell 13 3706-3719. 
Sharp DJ, Rogers GC \& Scholey JM 2000 Cytoplasmic dynein is required for poleward chromosome movement during mitosis in Drosophila early embryos. Nature Cell Biology 2 922-930.

Starr DA, Williams BC, Hays TS \& Goldberg ML 1998 ZW10 helps recruit dynactin and dynein to the kinetochore. Journal of Cell Biology 142 763-774.

Sun QY \& Schatten H 2006 Role of NuMA in vertebrate cells: review of an intriguing multifunctional protein. Frontiers in Bioscience $\mathbf{1 1}$ 1137-1146.

Tsurumi C, Hoffmann S, Geley S, Graeser R \& Polanski Z 2004 The spindle assembly checkpoint is not essential for CSF arrest of mouse oocytes. Journal of Cell Biology 167 1037-1050.

Vaisberg EA, Koonce MP \& McIntosh JR 1993 Cytoplasmic dynein plays a role in mammalian mitotic spindle formation. Journal of Cell Biology 123 849-858.

Vigneron S, Prieto S, Bernis C, Labbe JC, Castro A \& Lorca T 2004 Kinetochore localization of spindle checkpoint proteins: who controls whom. Molecular Biology of the Cell 15 4584-4596.

Wang WH \& Sun QY 2006 Meiotic spindle, spindle checkpoint and embryonic aneuploidy. Frontiers in Bioscience 11 620-636.
Wassmann K, Niault T \& Maro B 2003 Metaphase I arrest upon activation of the Mad2-dependent spindle checkpoint in mouse oocytes. Current Biology 13 1596-1608.

Weiss E \& Winey M 1996 The Saccharomyces cerevisian spindle pole body duplication gene MPS1 is part of a mitotic checkpoint. Journal of Cell Biology 132 111-123.

Zhang D, Ma W, Li YH, Hou Y, Li SW, Meng XQ, Sun XF, Sun QY \& Wang WH 2004 Intraoocyte localization of Mad2 and its relationship with kinetochores, microtubules and chromosomes in rat oocytes during meiosis. Biology of Reproduction 71 740-748.

Zhang D, Li M, Ma W, Hou Y, Li YH, Li SW, Sun QY \& Wang WH 2005 Localization of mitotic arrest deficient 1 (Mad1) in mouse oocytes during the first meiosis and its functions as a spindle checkpoint protein. Biology of Reproduction 72 58-68.

Received 3 March 2006

First decision 31 May 2006

Revised manuscript received 21 December 2006

Accepted 5 January 2007 